\title{
Modeling and Measurement Accuracy Enhancement of Flue Gas Flow Using Neural Networks
}

\author{
Haizhuang Kang, Qingping Yang, Member, IEEE, and Clive Butler
}

\begin{abstract}
This paper discusses the modeling of the flue gas flow in industrial ducts and stacks using artificial neural networks (ANN's). Based upon the individual velocity and other operating conditions, an ANN model has been developed for the measurement of the volume flow rate. The model has been validated by the experiment using a case-study power plant. The results have shown that the model can largely compensate for the nonrepresentativeness of a sampling location and, as a result, the measurement accuracy of the flue gas flow can be significantly improved.
\end{abstract}

Index Terms-Error analysis, gas flow measurement, measurement system data handling, modeling, neural network application.

\section{INTRODUCTION}

$\mathbf{C}$ URRENT measurements of emissions of pollutants to the environment have errors in excess of $20 \%$ at most thermal power stations and other industrial installations. This is mainly due to the low degree of representativeness of the gas samples collected, nonoptimum sensor location, and inaccuracies in the measurement of flue gas flows. In order to reduce the measurement error, it is important to optimize the sensor locations by means of the flue gas modeling.

This paper presents a novel method using neural networks to model the flue gas flow in industrial ducts and stacks. Several different designs including network architecture and training algorithms will be studied and compared.

\section{Measurement of the Volume Flow Rate}

\section{A. Using Manual Operation and ISO10780}

The volume flow rate of the flue gas flow in the industrial ducts and stacks are often calculated according to the ISO 10780, when a manual operation using Pitot tubes are employed.

The average Pitot tube pressure difference $\Delta \bar{p}$ is given as

$$
\Delta \bar{p}=\frac{1}{n^{2}}\left(\sum_{i=1}^{n} \sqrt{\Delta p_{i}}\right)
$$

where $\Delta p_{i}$ is the pressure difference at sampling point $i$. The unit is kilopascals. $n$ is the number of sampling points.

Manuscript received MAY 18, 1998; revised December 10, 1998. This work was supported by the EU-SMT Programme of the 4th Framework Programmes.

The authors are with the Department of Manufacturing and Engineering System, Brunel University, Uxbridge, Middlesex, UB8 3PH, U.K.

Publisher Item Identifier S 0018-9456(98)09879-9.

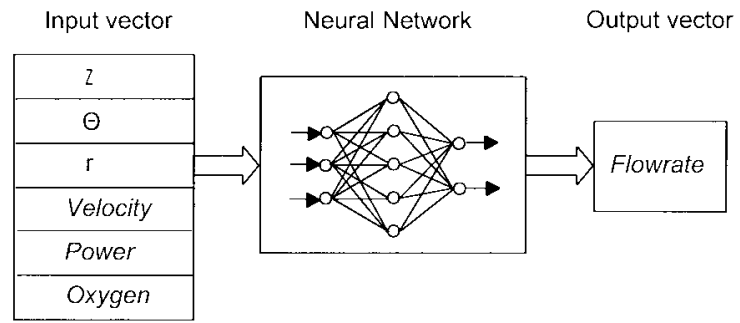

Fig. 1. Neural network model for predicting flue gas flow rate in industrial ducts and stacks.

The average gas velocity, $\bar{v}$, may be then expressed as

$$
\bar{v}=K C \sqrt{\frac{T_{s} \Delta \bar{p}}{p_{e} M_{s}}}
$$

where

$K \quad$ Pitot tube coefficient;

$C \quad$ Pitot tube constant $=129(\mathrm{~m} / \mathrm{s}) *[\mathrm{~kg} /(\mathrm{kmol} * \mathrm{~K})]^{1 / 2}$;

$T_{s}$ the average temperature at the section, in Kelvins;

$\Delta \bar{p}$ the average Pitot tube pressure difference in the section, in kilopascals;

$p_{e} \quad$ the absolute gas pressure, in kilopascals;

$M_{s}$ the molar mass of gas.

The volume flow rate at stack condition is defined as $q_{v s}=\bar{v} * A$, where

$\bar{v} \quad$ the average gas velocity in one section $(\mathrm{m} / \mathrm{s})$;

$A$ the cross-sectional area;

$q_{v s}$ the volume flow rate at stack condition $\left(\mathrm{m}^{3} / \mathrm{s}\right)$.

The volume flow rate at standard reference conditions (i.e., $0^{\circ} \mathrm{C}$ and $\left.101.3 \mathrm{KPa}\right) q_{v r}$ can be expressed as

$$
q_{v r}=q_{v s}\left(\frac{273}{T_{s}}\right)\left(\frac{P_{e}}{101.3}\right) \text {. }
$$

\section{B. Using Artificial Neural Networks with Manual Operation}

To be compatible with the ISO 10780, the proposed method will be based upon the manual operation using Pitot tubes. The measurements thus include static pressure, differential pressure, and temperature, taken at various sampling locations.

Since the measurement accuracy is significantly influenced by the nonrepresentativeness, which, in practice, is largely due to the instable gas flow in the sampled locations, it is very important to establish the relationship between the gas flows in a sampling location and a reference one which is stable and representative. 


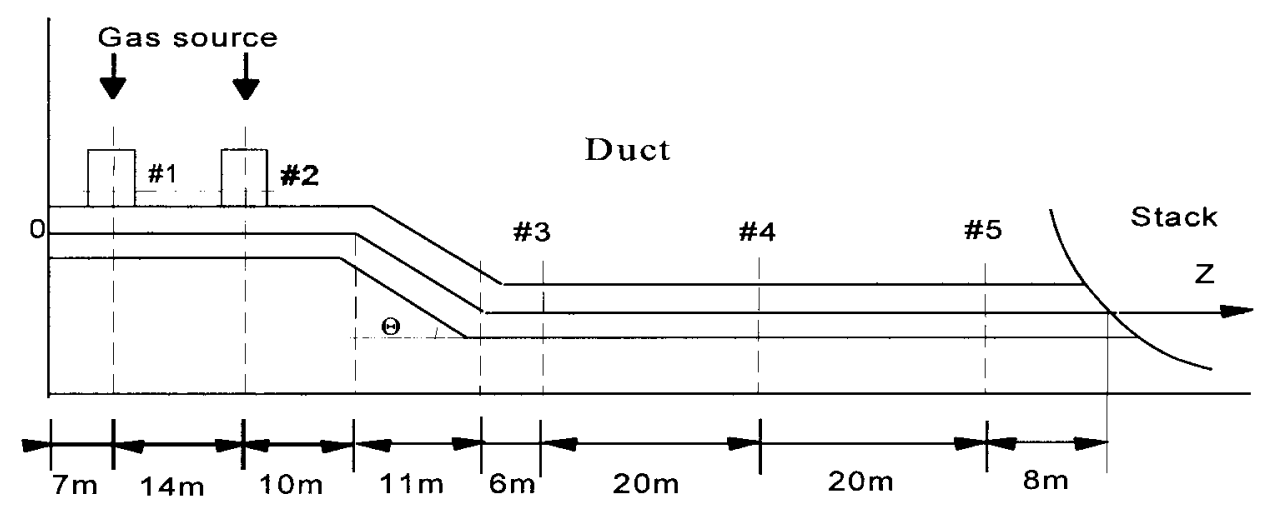

Fig. 2. Construction of the duct.

Because of the existence of the turbulence and disturbance in the gas flow, the relationship concerned is difficult to establish using a conventional method. The use of artificial neural networks is proposed due to its learning ability, as well as capacity for solving nonlinear and complicated problems, among other advantages. The trained neural network will then allow for a much improved prediction of the gas flow in a nonoptimized location, and can therefore compensate for the nonrepresentativeness of the gas samples, hence improving the measurement accuracy.

\section{Design of the Neural Network Model}

\section{A. Network Architecture}

The proposed neural network model is based on a threelayered feedforward neural network. As the volume flow rate is the product of average gas velocity and cross-sectional area of the sampling plane, the individual gas velocity is included in the input to the neural network. In order to establish the velocity profile and account for the spatial variations, the location of a sampling point is also represented in the model. In addition, the operating conditions (e.g., power and oxygen flow) are considered in the model.

As shown in Fig. 1, the input vector is $[z \theta r$ Velocity Oxygen Power], while the output vector is simply [Flowrate]. $z, \theta$, and $r$ in the input vector are the coordinates of the measurement point in a predefined coordinate system. The $z$ axis represents the location of the sampling plane along the centerline of the duct. Due to the circular shapes of the duct cross sections, the positions of sampling points in these sampling planes are represented as polar coordinates, $\theta$ and $r$. Velocity stands for individual velocity at the point $(z, \theta, r)$. At this moment, the output power in this unit is Power (MW) and the percentage of oxygen in the flue gas flow is Oxygen (\%). In the output vector, Flowrate is the volume flow rate in the sampling plane in the duct or stack corresponding to the individual velocity.

\section{B. Training and Testing}

The training of the neural network is based upon the Levenberg-Marquardt algorithm [3]. Typically, about half of the data in each sampling plane are randomly selected to train the neural network. After the training, two kinds of testing are then performed.

Test 1- Using the trained data set, i.e., those used in training

Test 2- Using the testing data set, i.e., those not used in training.

Because the initialization of the neural network is different each time, the training and testing are performed five times for each data set. The final results are the mean values of the five results. Test 2 normally gives a better indication of the generation ability of neural networks.

\section{EXPERIMENT}

\section{A. Experimental Setup}

The neural network model was validated with experiment based on a case-study plant, as shown in Fig. 2, where the duct presents the following geometry: the gas inlets occur at an angle of $900^{\circ}$ along two pipes measuring $5.5 \mathrm{~m}$ in diameter and $7 \mathrm{~m}$ in length. The flue gas flow is along a horizontal pipe of a diameter of $7.1 \mathrm{~m}$ for a distance of $31 \mathrm{~m}$ at the centerline. As from this point there is a horizontal elbow of $39^{\circ} 54^{\prime}$ measuring a total $13 \mathrm{~m}$ in length. Downstream from this elbow, another $52 \mathrm{~m}$ long duct of a diameter of $7.1 \mathrm{~m}$ leads directly into the stack.

\section{B. Sampling Locations}

According to the ISO 10780, sampling shall take place in a length of a straight duct with constant shape and crosssectional area, and, as far as possible, downstream from any obstruction which may cause a disturbance and produce a change in the direction of flow. The section of straight duct should be at least 7 hydraulic diameters long. Over the length of the straight section, locate the sampling plane at a distance of 5 hydraulic diameters from the inlet. If the sampling plane is to be located in a duct near the gas stream exit there should also be 5 hydraulic diameters (making a straight length of 10 hydraulic diameters). Based upon the above requirement, five sampling planes (\#1-\#5) were selected along the duct. In each sampling plane, four diameters were measured, with six sampling points on each radius (Fig. 3).

The measurements may be performed at different times in order to model the system under different operating conditions. 


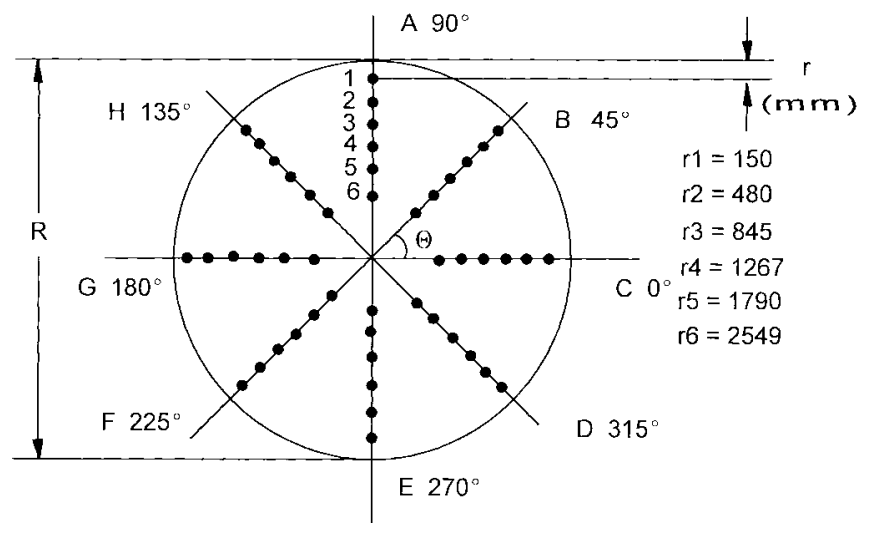

Fig. 3. Sampling plane.

TABLE I

Number of Pairs of Input-Output Vectors for Each Sampling Plane

\begin{tabular}{|l|l|l|l|l|l|}
\hline Sampling plane & $\# 1$ & $\# 2$ & $\# 3$ & $\# 4$ & $\# 5$ \\
\hline Number of pairs & 96 & 96 & 168 & 288 & 144 \\
\hline
\end{tabular}

TABLE II

FORMAT OF INPUT AND OUtPUT VECTOR

\begin{tabular}{|c|c|c|c|c|c|c|c|}
\hline \multirow{2}{*}{ Position } & \multicolumn{5}{|c|}{ Input vector } & Output vector \\
\cline { 2 - 8 } & $\begin{array}{c}\% \\
(\mathrm{~m})\end{array}$ & $\begin{array}{c}\theta \\
\left({ }^{\circ}\right)\end{array}$ & $\begin{array}{c}\mathrm{r} \\
(\mathrm{mm})\end{array}$ & $\begin{array}{c}\text { Velocity } \\
(\mathrm{m} / \mathrm{s})\end{array}$ & $\begin{array}{c}\text { Powcr } \\
(\mathrm{MW})\end{array}$ & $\begin{array}{c}\text { Oxygen } \\
(\%)\end{array}$ & $\begin{array}{c}\text { Flowrate in stack } \\
\left(\mathrm{m}^{3} / \mathrm{s}\right)\end{array}$ \\
\hline B1 & 66 & 45 & 150 & 21 & 357 & 6.25 & 529.8 \\
\hline B2 & 66 & 45 & 480 & 22.76 & 357 & 6.25 & 529.8 \\
\hline
\end{tabular}

As a pair of input and output vectors can be generated from each measurement, the total numbers of pairs of vectors in each sampling plane are shown in Table I.

\section{Data Format}

The data format is shown in Table II, with two examples from the measuring plane \#4. As the measurement was performed at the same time, these two samples have the same Power, Oxygen, and Flowrate.

\section{RESULTS AND DISCUSSIONS}

The data obtained from the experiment were preprocessed before the training and testing. The results will be discussed with two sampling planes \#4 and \#2.

The neural network had 5 neurons in the hidden layer and the training used an error goal of 0.01 with a maximum of 200 epochs. In sampling plane \#4, there were 288 samples available. Half of them, 140, were randomly selected for training and Test 1 , and the rest 148 for Test 2 (see Table III).

The results of Test 2 using 60 samples (from 141 through 200) are shown in Fig. 4 (solid line is the actual flow rate, dash line is the predicted flow rate). The prediction errors for each sample and distribution of prediction errors are shown in Figs. 5 and 6, respectively.

In sampling plane \#2, there were total 96 samples, 48 of them were randomly selected for training and Test 1 , the rest for Test 2. The results were given in Table IV.
TABLE III

Results of the Tests 1 and 2 in Sampling Plane \#4

\begin{tabular}{|c|c|c|c|}
\hline & & Test 1 & \\
\hline & mean absolute error $(\%)$ & standard devation $(\%)$ & max absolue error (\%) \\
\hline 1 & 0.026 & 0.030 & 0.080 \\
\hline 2 & 0.031 & 0.041 & 0.120 \\
\hline 3 & 0.036 & 0.017 & 0.070 \\
\hline 4 & 0.024 & 0.032 & 0.080 \\
\hline 5 & 0.000138 & 0.000147 & 0.000500 \\
\hline mean value & 0.023 & 0.024 & 0.070 \\
\hline & & & \\
\hline & & & \\
\hline & & Test 2 & \\
\hline & mean absolute error $(\%)$ & standard deviation $(\%)$ & max absolue error $(\%)$ \\
\hline 1 & 0.029 & 0.034 & 0.080 \\
\hline 2 & 0.033 & 0.045 & 0.200 \\
\hline 3 & 0.036 & 0.016 & 0.072 \\
\hline 4 & 0.029 & 0.037 & 0.150 \\
\hline 5 & 0.000153 & 0.000172 & 0.000800 \\
\hline mean value & 0.025 & 0.026 & 0.101 \\
\hline
\end{tabular}

TABLE IV

Results of the Tests 1 and 2 in Sampling Plane \#2

\begin{tabular}{|c|c|c|c|}
\hline & & Test 1 & \\
\hline & mean absolute error (\%) & standard devation (\%) & max absolue error (\%) \\
\hline 1 & 0.18 & 0.26 & 0.60 \\
\hline 2 & 0.18 & 0.25 & 0.50 \\
\hline 3 & 0.17 & 0.25 & 0.60 \\
\hline 4 & 0.20 & 0.27 & 0.60 \\
\hline 5 & 0.18 & 0.27 & 0.60 \\
\hline mean value & 0.18 & 0.26 & $\mathbf{0 . 5 8}$ \\
\hline & & & \\
\hline & & & \\
\hline & & Test 2 & 0.90 \\
\hline & mean absolute error (\%) & standard devation (\%) & max absolue error (\%) \\
\hline 1 & 0.38 & 0.47 & 5.80 \\
\hline 2 & 0.62 & 1.15 & 45.00 \\
\hline 3 & 3.38 & 9.67 & 2.00 \\
\hline 4 & 0.51 & 0.65 & 3.20 \\
\hline 5 & 0.46 & 0.70 & 11.38 \\
\hline mean value & 1.07 & $\mathbf{2 . 5 3}$ & \\
\hline
\end{tabular}

From Table IV, it can be seen that as sampling plane \#2 is very close to the inlets of the gas flow and the elbow, the flow in this plane was not as stable as that in sampling plane \#4, and some large prediction errors have occurred. Sampling plane \#4 as the sensor location is therefore much better than sampling plane \#2. This conclusion also agrees with the ISO 10780. The results of Test 2 using the 48 testing samples are shown in Fig. 7 (solid line is the actual flow rate, dash line is the predicted flow rate). The prediction errors for each sample and distribution of prediction errors are given in Figs. 8 and 9.

\section{CONCLUSIONS}

The use of artificial neural networks for the modeling of the flue gas flow in industrial stacks and ducts has been discussed, together with the results from a case-study power plant. The following conclusions may be drawn from the above discussions.

1) Using the neural network model, the volume flow rate of the flue gas flow in the ducts and stacks can be predicted with the individual velocity, plus Power and Oxygen.

2) As a result, the neural network model is able to compensate for the nonrepresentativeness of the gas samples, 


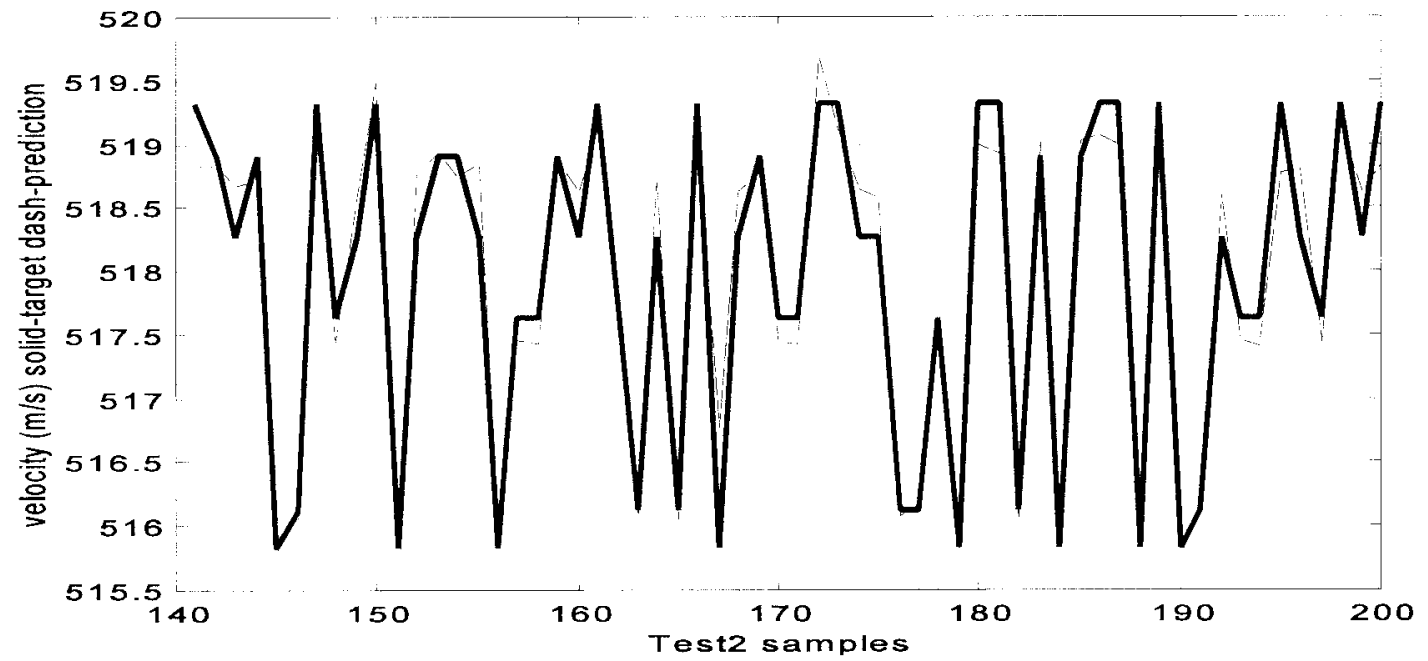

Fig. 4. Part of Test 2 results in sampling plane \#4.

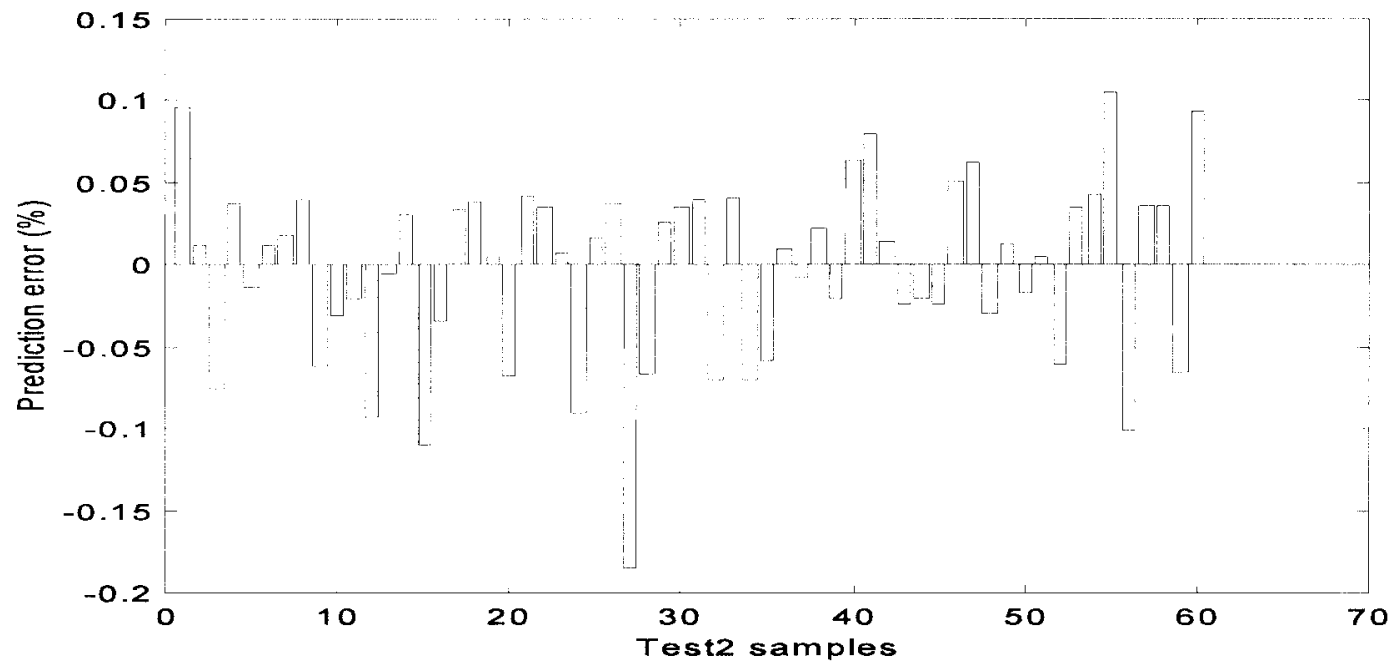

Fig. 5. Prediction errors for each testing sample in sampling plane \#4.

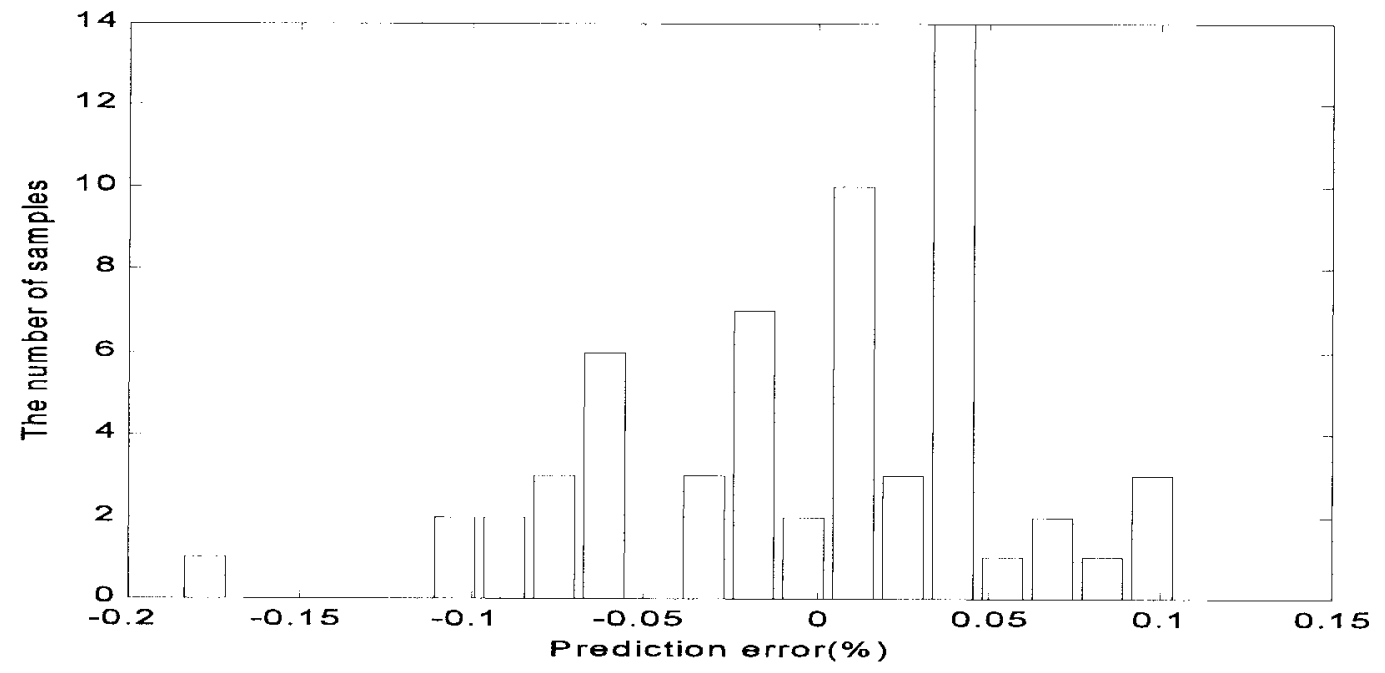

Fig. 6. Distribution of prediction errors in sampling plane \#4. 


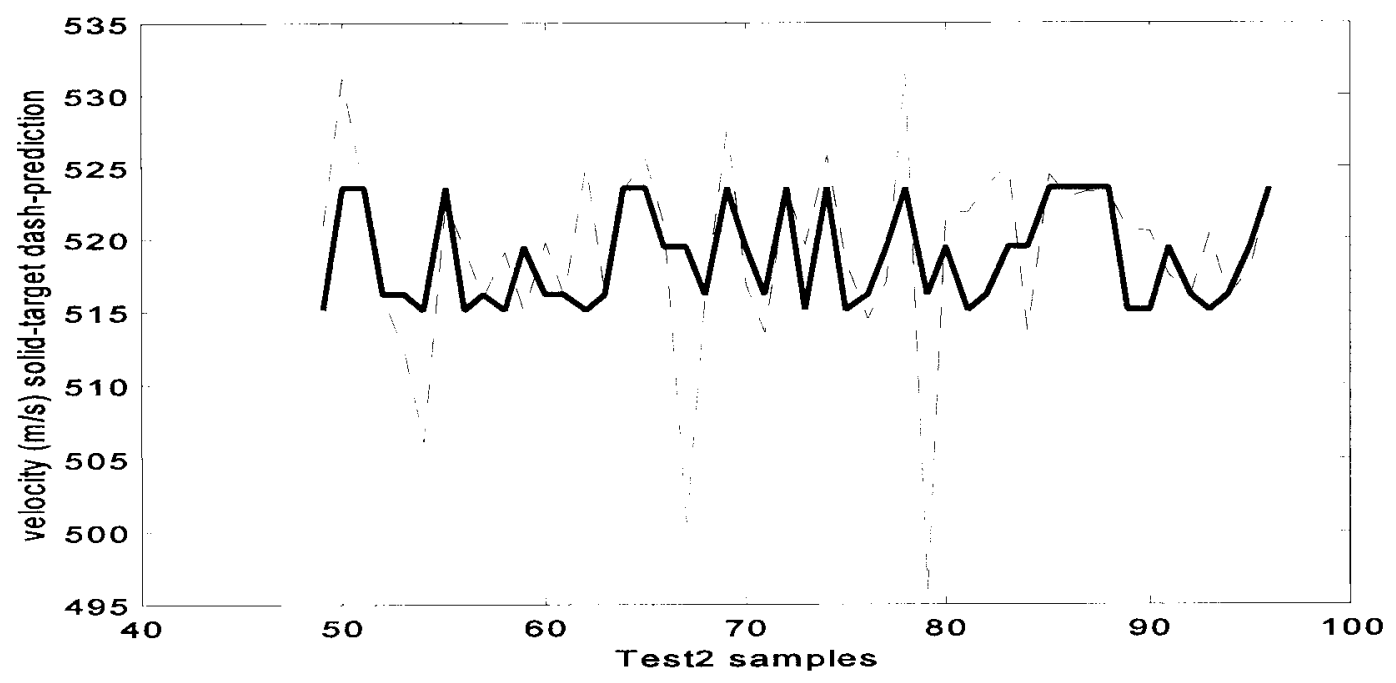

Fig. 7. Test 2 results in sampling plane \#2.

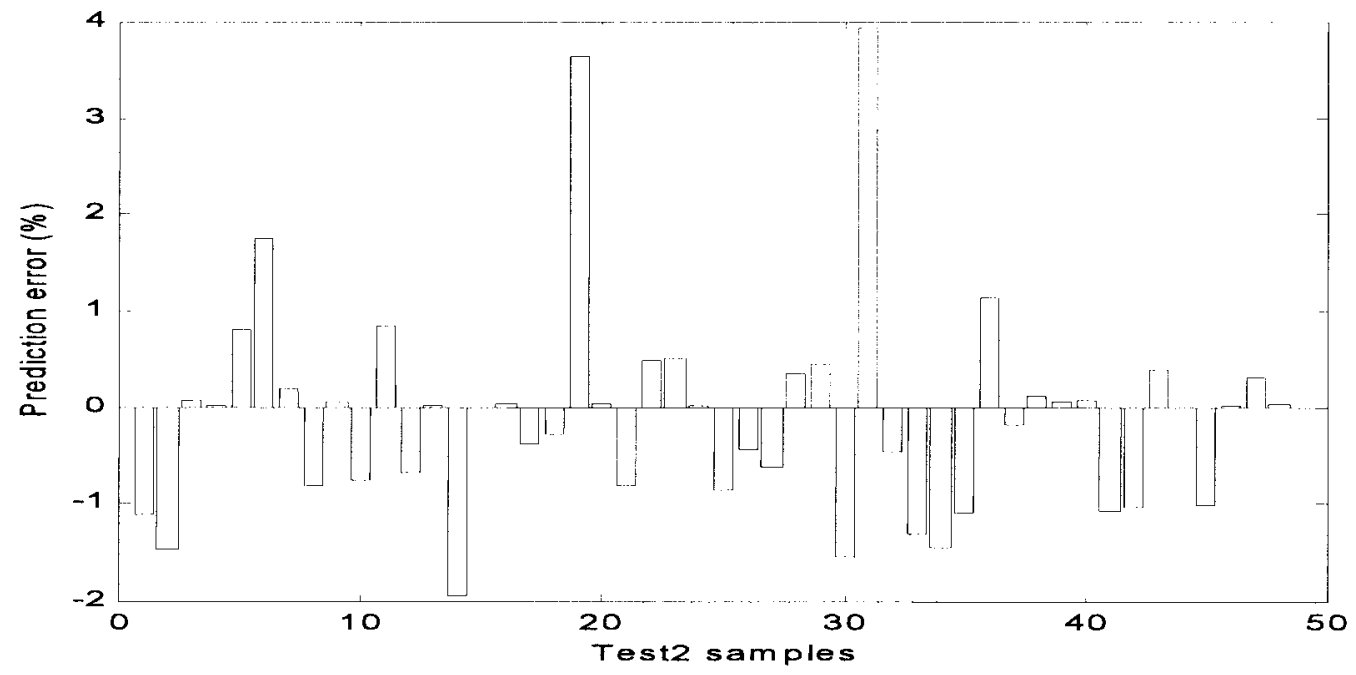

Fig. 8. Prediction errors for each testing sample in sampling plane \#2.

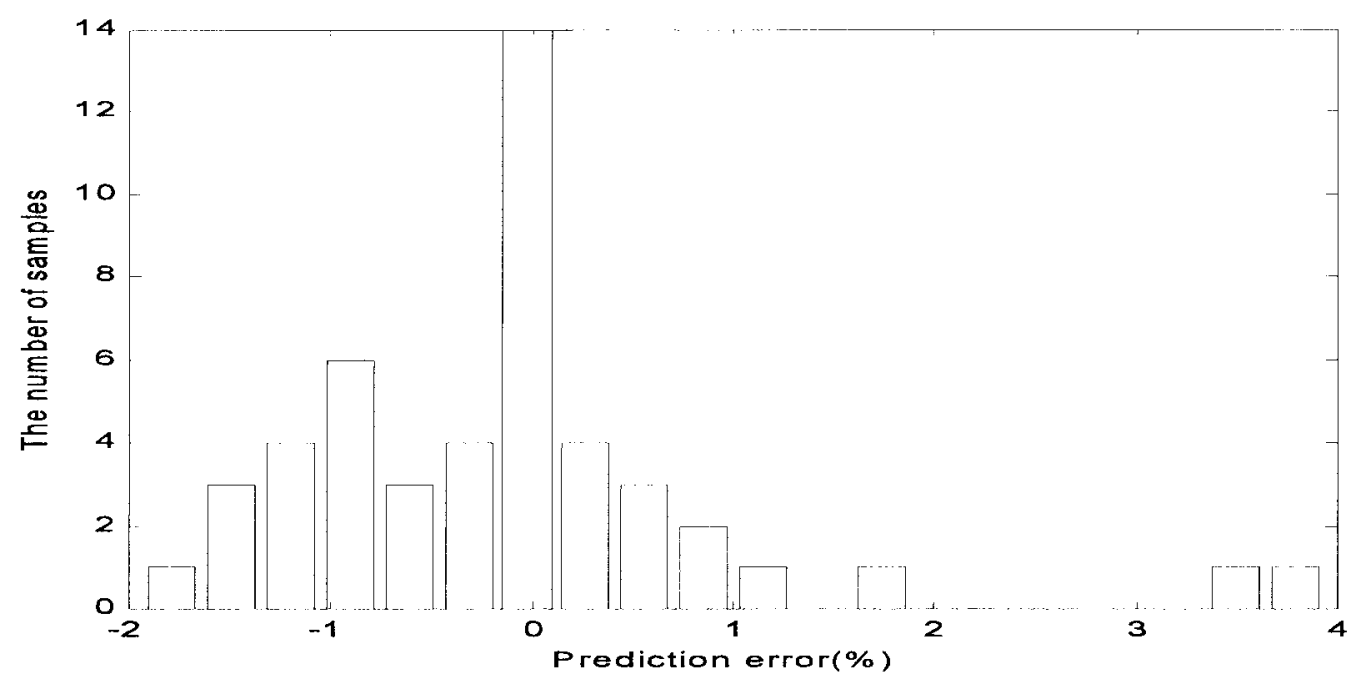

Fig. 9. Distribution of prediction errors in sampling plane \#2. 
and hence improve the measurement accuracy of the gas flow.

3) The sensor location is an important factor in the measurement of the flue gas flow, and can sometimes cause large measurement errors. It is necessary to optimize the sensor location, in order to achieve better measurement accuracy.

Since the above model only used individual velocity, large errors may result from the turbulence and disturbance in the ducts and stacks. A more robust model with more information about the velocity profiles will considerably reduce the risk of producing large errors and improve the neural network performance. The optimization of sensor locations and the more robust models will be reported in the future.

\section{REFERENCES}

[1] OLASIS project report, KEMA, "Uncertainty in flue gas flow measurements," SMT4-CT95-2023/KE-04T, 1996.

[2] ISO 10780, "Stationary source emissions," in Measurement of Velocity and Volume Flowrate of Gas Streams in Duct, 1994

[3] K. Kemuth and M. Beale, Neural Network ToolBox, The Math Works Inc, MA, 1994

[4] G. W. Irwin, K. Warwick, and K. J. Hunt, Neural Network Applications in Control. Herts, U.K. IEE, 1995.

[5] OLASIS project report, "As Ponts thermal power plant model validation," SMT4-CT95-2023/TE-21T, 1997.

[6] J. A. Roberson and C. T. Crowe, Engineering Fluid Mechanics, 6th ed. New York: Wiley, 1997.

[7] OLASIS project report, "Neural network application for gas flow measurement," SMT4-CT95-2023/BU06T, Apr. 1997.

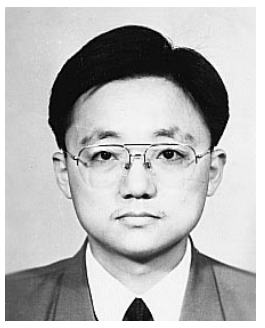

Haizhuang Kang received the B.Sc. degree in radio communication from Xidian University, Xi'an, China in 1987. In 1994 he received the M.Sc. degree in communication and electronic system at the same university.

He was an Assistant Engineer and head of Communication Laboratory at Xidian University and then worked for Great Wall Computer Company as an engineer and head of communication equipment factory in Shenzhen, China. He is now a Ph.D. student at Brunel University, Uxbridge, Middx, U.K. His current research interests include advanced sensors and measurement system, measurement instrument, neural network application, image processing system, and military communication networks.

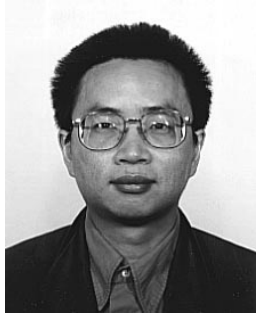

Qingping Yang (M'97) received the Diploma in instrumentation and measurement from Chengdu Aeronautical Polytechnic, Chengdu, China in 1983 , and the Ph.D. degree from Brunel University, Uxbridge, Middx, U.K., in 1992.

He was then an Assistant Engineer, Deputy Head of the Department of Measurement and Testing in the Aircraft Structural Strength Research Institute (Ministry of Aerospace), Shaanxi Province, China He was awarded a scholarship to study at Brunel University from 1988 to 1992 . His current research interests include dimensional metrology, advanced sensors and transducers, and virtual instrumentation and measurement science. He has published more then 30 papers in these areas.

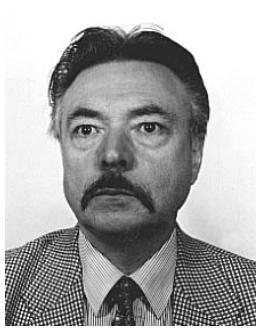

Clive Butler received the B.Sc. degree in physics from the University of Manchester, U.K., in 1963 and the M.Sc. and Ph.D. degrees from Imperial College, London, U.K., in 1968.

He has held a number of industrial posts including Chief Physicist, OMT Ltd, and Director, Metronic Technology Ltd and Digital Metrology Ltd. Previously Director of Research at Watford College of Technology. He is now Professor at Brunel University, Uxbridge, Middx, U.K. His current research interests are in dimensional metrology and quality systems. He has presented more than 60 papers in applied optics, metrology, quality management, and printing technology.

Dr. Butler is a fellow of the Royal Society of Arts and member of the Institute of Quality Assurance. 